\title{
Universiteit
}

Leiden

The Netherlands

\section{Multiple order parameter configurations in superconductor/ferromagnet multilayers}

Kushnir, V.N.; Prischepa, S.L.; Cirillo, C.; Vecchione, A.; Attanasio, C.; Kupriyanov, M.Yu.; Aarts, J.

\section{Citation}

Kushnir, V. N., Prischepa, S. L., Cirillo, C., Vecchione, A., Attanasio, C., Kupriyanov, M. Y., \& Aarts, J. (2011). Multiple order parameter configurations in superconductor/ferromagnet multilayers. Physical Review B, 84, 214512. doi:10.1103/PhysRevB.84.214512

Version: $\quad$ Not Applicable (or Unknown)

License: $\quad$ Leiden University Non-exclusive license

Downloaded from: https://hdl.handle.net/1887/45118

Note: To cite this publication please use the final published version (if applicable). 


\title{
Multiple order parameter configurations in superconductor/ferromagnet multilayers
}

\author{
V. N. Kushnir, ${ }^{1}$ S. L. Prischepa, ${ }^{1}$ C. Cirillo,${ }^{2}$ A. Vecchione, ${ }^{2}$ C. Attanasio, ${ }^{2}$ M. Yu. Kupriyanov, ${ }^{3}$ and J. Aarts ${ }^{4}$ \\ ${ }^{1}$ State University of Informatics and RadioElectronics, P. Browka 6, Minsk 220013, Belarus \\ ${ }^{2}$ CNR-SPIN Salerno and Dipartimento di Fisica “E.R. Caianiello”, Università degli Studi di Salerno, Fisciano (Sa) IT-84084, Italy \\ ${ }^{3}$ Nuclear Physics Institute, Moscow State University, Moscow 119992, Russia \\ ${ }^{4}$ Kamerlingh Onnes Laboratory, Leiden University, P.O. Box 9504, NL-2300 RA Leiden, The Netherlands \\ (Received 13 June 2011; revised manuscript received 10 October 2011; published 7 December 2011)
}

\begin{abstract}
The coupling of two superconductors (S) through a ferromagnet (F) can lead to either a zero- or a $\pi$-phase difference between the superconducting banks. Most research in this area is performed on trilayer S/F/S film structures, in which two-order parameter configurations are possible. Increasing the number of layers and junctions leads to a larger number of possible configurations with, in principle, different properties such as the superconducting transition temperature $T_{c}$. Here we study the behavior of a series of multilayers made of superconducting $\mathrm{Nb}$ and ferromagnetic $\mathrm{Pd}_{81} \mathrm{Ni}_{19}$. We find that for the individual layer thicknesses used, the transition width $\Delta T_{c}$ increases with increasing number of bilayers in the multilayer, in a well-defined manner. That the broadening is not simply due to increased disorder in the larger stacks, it is shown from x-ray diffraction, which finds very sharp interfaces for all samples; and from the effect of the magnetic field on the transition, which shows a considerable sharpening. We can make a connection with the various order parameter configurations using a matrix formulation of quasiclassical theory based on the Usadel equations and show that these different configurations take part in the Josephson networks, which are building up in the transition to the superconducting state.
\end{abstract}

DOI: 10.1103/PhysRevB.84.214512

PACS number(s): 74.78.Fk, 74.45.+c

\section{INTRODUCTION}

When combining a superconducting (S) and a ferromagnetic (F) thin film, it is well known that the superconducting correlations induced in the ferromagnet are spatially inhomogeneous. ${ }^{1}$ In SFS junctions, by choosing the appropriate value for the F-layer thickness $d_{F}$, this can lead to a socalled $\pi$ state, in which the phase of the superconducting order parameter changes over $\pi$ when going from one side of the junction to the other. This is by now a well-established effect, with experimental consequences such as strong variations of the junction critical current as a function of temperature, ${ }^{2}$ or spontaneous currents occurring in a superconducting ring with an SFS junction. ${ }^{3}$ Obviously the SFS trilayer can only have two ground states: one without phase change, and one with a phase change (equivalent to a sign change or a node) in the pair amplitude. The two states have a different critical temperature $T_{c}$, leading to the well-known $T_{c}$ oscillations as function of $d_{F} .{ }^{4}$ When more junctions are added to the stack, the number of possible modes can be expressed in terms of a primary building block $\frac{1}{2} d_{F} / d_{S} / \frac{1}{2} d_{F}$ (with $d_{S}$ the $S$-layer thickness) as $N_{\text {tri }}+1$, where $N_{\text {tri }}$ is the number of blocks. More than one node is now possible, which distinguishes the $\mathrm{S} / \mathrm{F}$ case from multilayers with normal metals, and S/F multilayers from an SFS trilayer. Each mode can be characterized by its own critical temperature. A first attempt to clarify such new issues was recently published. ${ }^{5}$ Incidentally, for $T_{c}$ the well-known limit to the problem is the infinite multilayer (IM) with periodic boundary conditions. With this approach, calculations were for instance made of the upper critical field behavior in $\mathrm{S} / \mathrm{N}$ superlattices, ${ }^{6}$ while for the $\mathrm{F}$ case it actually led to the prediction of oscillatory $T_{c}\left(d_{F}\right){ }^{7,8}$ On general grounds, the infinite multilayer $T_{c}$ with a symmetrical periodic solution furnishes the upper limit for $T_{c}$ of a system with a finite number of blocks.
In this work we compare the nucleation of superconductivity in samples with different symmetries, where different modes can play a weaker or a stronger role. For the preparation we use weakly ferromagnetic $\mathrm{Pd}_{81} \mathrm{Ni}_{19}$ and superconducting $\mathrm{Nb}$, and choose a value $d_{F}$ somewhat lower than the value where the $0-\pi$ transition in a simple $S / F / S$ junction is expected. ${ }^{9}$ In particular we compare samples with fixed $d_{F}, d_{S}$, and IM symmetry, consisting of $N_{\text {tri }}$ building blocks defined above, with samples consisting of a starting $\mathrm{F}$ layer and $N_{\text {bi }}(\mathrm{F} / \mathrm{S})$ bilayers notation $\left.\left[\mathrm{F} / N_{\mathrm{bi}}(\mathrm{S} / \mathrm{F})\right]\right\}$. Such samples we call asymmetric in the sense that they do not possess the IM symmetry, even though they possess a mirror plane. A sketch of both types of multilayers is given in Fig. 1. We find that this difference in symmetry (where actually only the outer layers have different thicknesses) has clearly observable effects on the nucleation, both seen in the width of the resistive transition $\Delta T_{c}$ and in its shape. For the symmetric samples, $\Delta T_{c}$ is small $(\approx 50 \mathrm{mK})$ and hardly changes with increasing $N_{\text {tri }}$. For the asymmetric samples, $\Delta T_{c}$ gradually increases until a maximum width of around $2 \mathrm{~K}$ is reached around $N_{\mathrm{bi}}=9$, after which it becomes constant. We also find the occurrence of steps in the resistance. In one particular sample with $N_{\mathrm{bi}}=9$, the number of steps even comes close to the number of possible modes $N_{\text {bi }}$. However, in a magnetic field the broad transitions sharpen up again. Next we demonstrate that both the increasing $\Delta T_{c}$ as well as its final value can be directly connected to the increasing number of modes. For this we use a matrix formulation of the quasiclassical theory on basis of the Usadel equation. We use measurements on bilayer building blocks as input parameters, calculate the $T_{c}$ values of the different modes, and extract in this way a highest and lowest value of $T_{c}$ for a multilayer, which can be connected to the measured $\Delta T_{c}$. The agreement between theory and experiment is very good, indicating that the modes play a role in the phase transition. We discuss this in terms of networks of Josephson junctions 


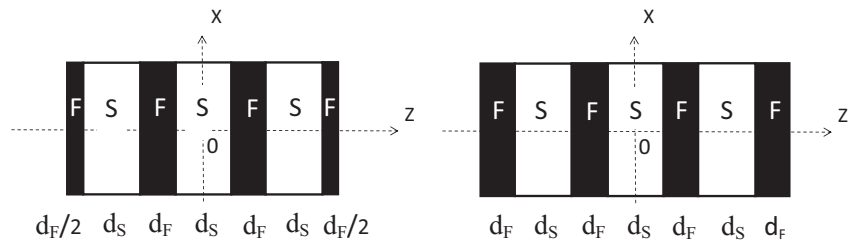

FIG. 1. Left: symmetric multilayer consisting of building blocks $\frac{1}{2} d_{F} / d_{S} / \frac{1}{2} d_{F}$ (infinite multilayer symmetry). Right: asymmetric multilayer consisting of a starting $\mathrm{F}$ layer and $N_{\mathrm{bi}}(\mathrm{F} / \mathrm{S})$ bilayers. In both cases the mirror axis of the multilayers is indicated.

which are formed throughout the transition. ${ }^{10}$ Moreover, we demonstrate that this network formation is reinforced for $d_{F}$ values close to the $0-\pi$ transition.

The paper is organized as follows. First we describe sample preparation and characterization by $\mathrm{x}$-ray reflectometry in order to demonstrate the structural integrity of the samples. Next we give the results of measurements of the resistance $R$ as function of temperature $T$ for both symmetric and asymmetric multilayers around the superconducting transition, and we show measurements of the dependence of $R$ on an applied magnetic field $H_{a}$ at a fixed temperature below $T_{c}$ for asymmetric samples. Then we develop the theoretical framework and we give a detailed example of the evolution of order parameter configurations as function of F-layer thickness for a fictitious five-bilayer asymmetric sample. That allows a discussion of our results in terms of such configurations, and the demonstration that the increasing transition width is connected to the increasing number of possible modes. We end with another experimental example of $T_{c}$ broadening in asymmetric multilayers using blocks of $\mathrm{Cu}_{41} \mathrm{Ni}_{59} / \mathrm{Nb}$.

\section{EXPERIMENT}

The samples consist of $\mathrm{Si} /\left[\mathrm{Pd}_{81} \mathrm{Ni}_{19} / N_{\mathrm{bi}}\left(\mathrm{Nb} / \mathrm{Pd}_{81} \mathrm{Ni}_{19}\right)\right]$ and were grown on $\mathrm{Si}(100)$ substrates by diode sputtering in an ultrahigh vacuum system as described in Ref. 9. Three series were grown; two asymmetric ones called MA $n$ and $\mathrm{MB} n$, with $n$ the number of bilayers, which runs from 5 to 9 for MA and from 3 to 14 for MB; and one symmetric set called MS $n$ consisting of blocks of $\frac{1}{2} d_{F} / d_{S} / \frac{1}{2} d_{F}$ and $n$ running from 1 to 9 . The layer thicknesses nominally grown were $d_{S}=18.7 \mathrm{~nm}(\mathrm{MA} n)$ or $16.0 \mathrm{~nm}(\mathrm{MB} n, \mathrm{MS} n)$, and $d_{F}=$ $2.2 \mathrm{~nm}$ (all). The latter is somewhat lower than the value of $3.1 \mathrm{~nm}$ where the $0-\pi$ transition in a simple $\mathrm{S} / \mathrm{F} / \mathrm{S}$ junction is expected, but the results show that this deviation is not critical. The number of $3.1 \mathrm{~nm}$ needs some explanation, which will be given in Appendix A. Magnetization measurements at $10 \mathrm{~K}$ confirmed the presence of ferromagnetism. The resistance $R$ was measured as function of temperature $T$ using a four-probe technique, with indium contact pads put in line on the top of the (unstructured) samples. The structural properties were characterized by $x$-ray reflectometry (XRR). Since the structural quality is important, we show in Fig. 2 the result for the symmetric sample MS2 and the asymmetric sample MA9. The data were fitted using the Parrat and Nevot-Croce recursion relation, which takes into account the electron density height fluctuations at the interface. ${ }^{11,12}$ Within the fit procedure the roughness of each single layer was
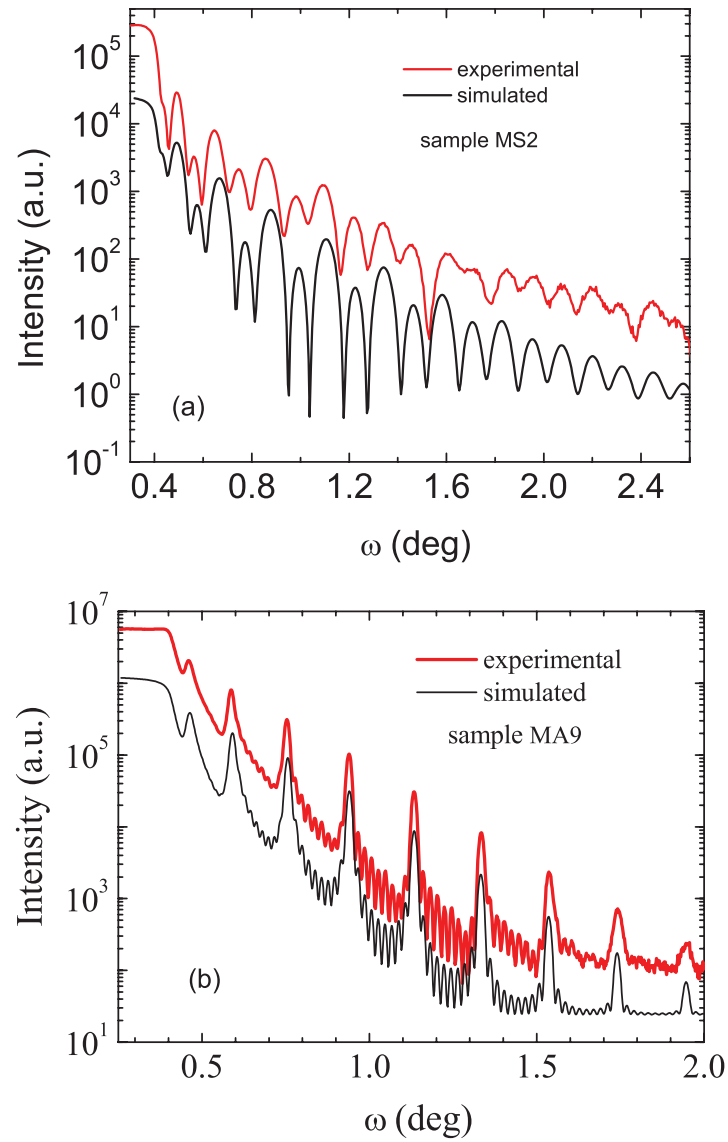

FIG. 2. (Color online) Measured x-ray reflectometry spectrum (upper) and numerical simulations (lower; shifted downward for clarity) for (a) sample MS2 and (b) sample MA9.

supposed to be independent on the roughness of other layers (the case of uncorrelated roughness ${ }^{13}$ ). Fitted values for the layer thicknesses came out very closely to the nominal values. For MS2, $d_{\mathrm{PdNi}}^{\mathrm{ext}}=1.0 \mathrm{~nm}, d_{\mathrm{PdNi}}=2.0 \mathrm{~nm}, d_{\mathrm{Nb}}=15.8 \mathrm{~nm}$; for MA9, the values were 2.2, 2.2, and $18.7 \mathrm{~nm}$, respectively. In the framework of this model we deduce that the thickness of $\mathrm{Nb}$ and PdNi layers is constant in the multilayer structures, while the values of the root-mean-square roughness are in general smaller for the internal layers $(0.4-0.8 \mathrm{~nm})$ and higher for the bottom and the capping layers $(0.8-1.1 \mathrm{~nm})$. Samples $\mathrm{MB} n$ have the same nominal characteristics, but their $T_{c} \mathrm{~s}$ were somewhat lower, probably due to slightly thinner $\mathrm{Nb}$ layers.

\section{RESULTS}

\section{A. $R(T)$ of symmetric and asymmetric multilayers}

Figures 3(a) and 3(b) show $R / R_{n}(T)$, the resistance normalized to a value just above $T_{c}$ for several $\mathrm{MS} n$ and $\mathrm{MB} n$ samples, respectively. For the MS series, the transitions are very sharp, no more than $50 \mathrm{mK}$ wide. Most of them show a small step which lowers $R$ by no more than $10 \%$, followed by the main transition.

For the MB series the transitions are broader from the start, and at higher $n$ the width is of the order of $1.5 \mathrm{~K}$. For all samples, zero resistance is reached in a final sharp drop at nearly the same temperature. It is remarkable that this 

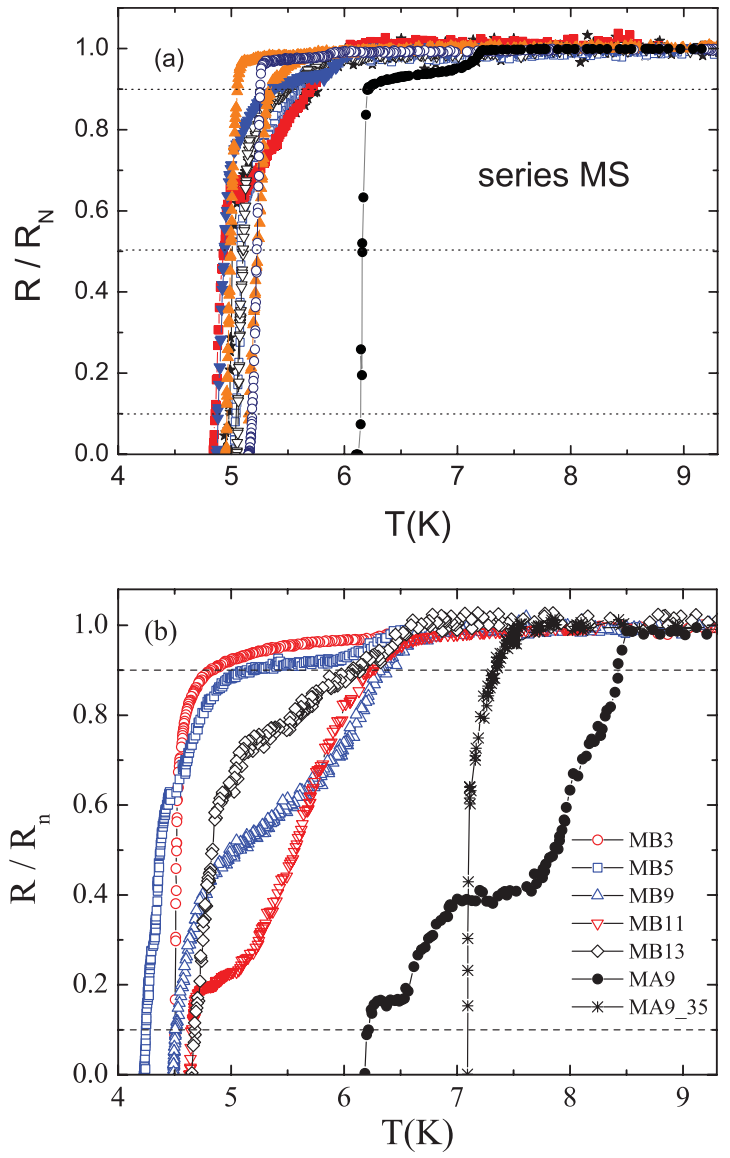

FIG. 3. (Color online) Normalized resistive transitions for different $\mathrm{Nb} / \mathrm{PdNi}$ multilayers. (a) From the series MSn. The sample with the highest $T_{c}$ has a single $\mathrm{S}$ layer; the other ones have $n$ from 2 to 9 . (b) From the series MB, as indicated. Also shown is the transition for sample MA9 and the sample MA9-35 with the middle S layer having a thickness of $35 \mathrm{~nm}$ (see text).

difference is due to only changing the outer $(\mathrm{F})$ layers from a thickness of $1 \mathrm{~nm}$ to a thickness of $2 \mathrm{~nm}$. In the broad transitions, hints can be seen of more steps. To make this more clear, also shown in Fig. 3(b) is $R(T)$ for MA9, which is the best example of such a multistep transition. Indications of similar broadening of $R(T)$ curves of $\mathrm{S} / \mathrm{F}$ hybrids can be found, ${ }^{14-17}$ but to our knowledge were never explicitly investigated. One more curve is shown in Fig. 3(b) of a sample similar to MA9, but now with a central (S) layer of $35 \mathrm{~nm}$. This sample again shows a very sharp transition. In Fig. 4 we show the variation of the transition width $\Delta T_{c}=T\left(0.9 R_{n}\right)-$ $T\left(0.1 R_{n}\right)$ for the samples $\mathrm{MB} n$, which we consider the central result of our work. The plot shows that the zero-resistance value is reached around 4.4-4.5 $\mathrm{K}$ and does not vary much through the series, but that the transition width increases and becomes constant again above $N_{\mathrm{bi}}=9$, where it is $1.5 \mathrm{~K}$.

\section{B. The field dependence of sample MA9}

In order to better understand the origin of the transition widening in asymmetric multilayers, best shown by sample MA9, we also measured $R$ both as function of $T$ in a fixed magnetic field $H_{a}$, oriented parallel to the layers; and as function of $H_{a}$ for fixed $T$, as shown in Figs. 5(a) and 5(b).

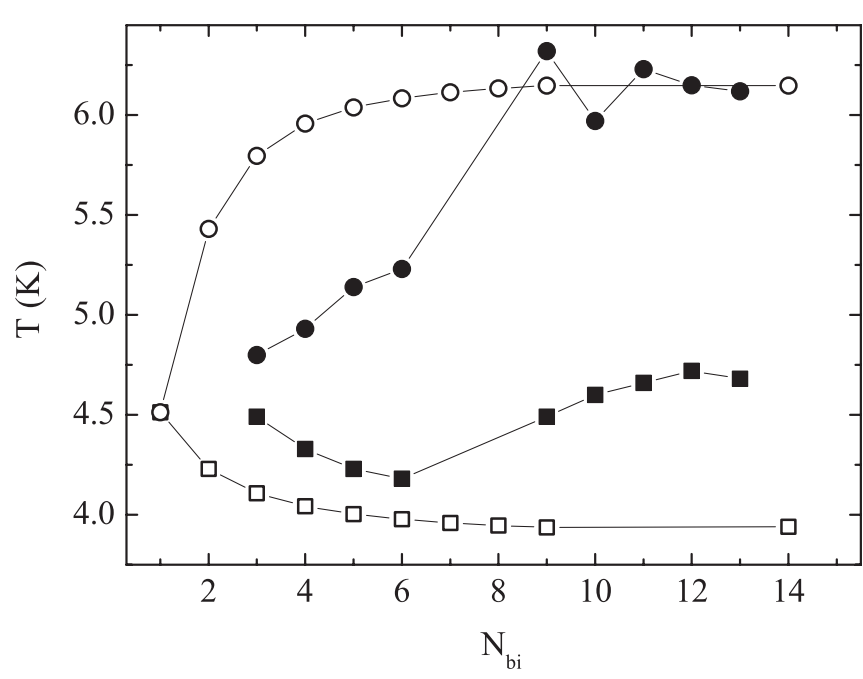

FIG. 4. The transition width for the samples $\mathrm{MB} n$ vs number of bilayers $N_{\text {bi }}$. Experiment : shown are the temperatures for $0.9 R_{n}$ (closed circles) and $0.1 R_{n}$ (closed squares), with $R_{n}$ the normalized resistance. Theory: open squares (circles) correspond to the maximum (zero) node state.

The curves in fixed field show a shift, but the $R(T)$ behavior is essentially unchanged from the zero-field behavior, with multiple steps visible. The curves at fixed temperature behave differently and sharpen up appreciably in higher applied fields, from about $1 \mathrm{~T}$ to less than $0.2 \mathrm{~T}$.

\section{THEORETICAL FRAMEWORK}

\section{A. Formulation of the model}

In order to explain these observations, a model was developed to calculate $T_{c}$ of the different order parameter configurations in finite multilayers. The onset of the critical state, in the diffusive limit and neglecting paramagnetic and spin-orbit effects, is described by a system of linearized Usadel equations $^{1,18}$ for the $S$ and $\mathrm{F}$ layers (taking $k_{B}=\hbar=1$ ):

$$
\begin{gathered}
-\pi T_{c S} \xi_{S}^{2} F_{n}^{\prime \prime}(z)+\left|\omega_{n}\right| F_{n}(z)=\pi T \lambda \sum_{\omega_{m} \mid \leqslant \omega_{D}} F_{m}(z), \\
-\pi T_{c S}\left(\xi_{F}^{*}\right)^{2} F_{n}^{\prime \prime}(z)+\left[\left|\omega_{n}\right|+i E_{\mathrm{ex}} \operatorname{sgn}\left(\omega_{n}\right)\right] F_{n}(z)=0 .
\end{gathered}
$$

Here, $T_{c S}$ is the bulk critical temperature of the superconductor, $\omega_{n}=\pi T(2 n+1)$ are the Matsubara frequencies $[n=0$, $\left.\pm 1, \ldots, n_{D}(T)\right]$, with $n_{D}(T)$ the integer part of the expression $\left(\omega_{D} / 2 \pi T-0.5\right)$ and $\omega_{D}$ the Debye frequency, $\lambda$ is the effective electron-electron interaction constant, $F_{n}(z)$ are the Usadel anomalous Green functions, and $E_{\mathrm{ex}}$ is the exchange field energy. The $z$ axis is taken perpendicular to the layers, while the $x y$ plane at $z=0$ coincides with the mirror plane of the sample. Furthermore, $\xi_{S}, \xi_{F}^{*}$ are the dirty limit coherence lengths in the $\mathrm{S}(\mathrm{F})$ metal, given by $\sqrt{D_{S, F} /\left(2 \pi T_{c S}\right)}$, with $D_{S, F}$ the diffusion coefficients in $\mathrm{S}(\mathrm{F})$. These equations are supplemented by matching and boundary conditions: ${ }^{19}$

$$
\begin{gathered}
\rho^{-1}\left(z_{i}^{+}\right) F_{n}^{\prime}\left(z_{i}^{+}\right)=\rho^{-1}\left(z_{i}^{-}\right) F_{n}^{\prime}\left(z_{i}^{-}\right), \\
F_{n}\left(z_{i}^{+}\right)=F_{n}\left(z_{i}^{-}\right)+\gamma_{b} \xi_{S} \frac{\rho_{F}}{\rho\left(z_{i}^{-}\right)} F_{n}^{\prime}\left(z_{i}^{-}\right), \\
F_{n}^{\prime}(-L / 2)=F_{n}^{\prime}(L / 2)=0 .
\end{gathered}
$$



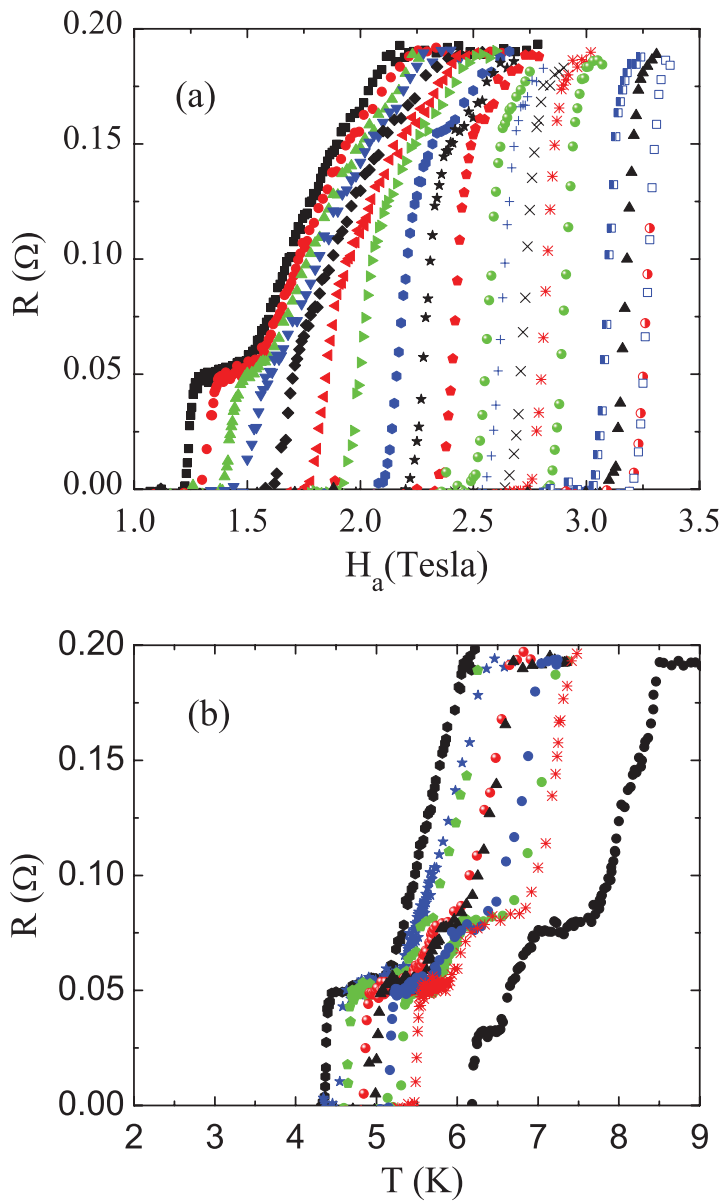

FIG. 5. (Color online) Field dependence of the resistive transition of sample MA9. (a) Resistance $R$ as function of in-plane applied field $H_{a}$ at temperatures (left to right) $T=4.25,4.14,4.05,3.94,3.82$, $3.68,3.54,3.36,3.25,3.10,2.94,2.87,2.74,2.63,2.51,2.24,2.13$, 1.99 , and $1.96 \mathrm{~K}$. (b) $R(T)$ for fields (right to left) $H_{a}=0,0.4,0.5$, $0.6,0.7,0.8,0.9,1.0$, and $1.1 \mathrm{~T}$.

Here $L$ is the overall thickness of the multilayer, $z_{i}(i=$ $\left.1,2, \ldots, 2 N_{\mathrm{bi}}\right)$ are the $z$ coordinates of the interfaces, $z_{i}^{ \pm} \equiv$ $z_{i} \pm 0$, and $\rho(z)=\rho_{F, S}$ for $z$ in $\mathrm{F}$, S, with $\rho_{F, S}$ the lowtemperature specific resistance of the $\mathrm{F}, \mathrm{S}$ layer. The parameter $\gamma_{b}$ is defined as $\left(2 \ell_{F}\right) /\left(3 t_{b} \xi_{F}^{*}\right)$, with $\ell_{F}$ the electron mean free path in the ferromagnet and $0<t_{b}<\infty$ the transparency parameter. For solving the set of equations the matrix method is used. ${ }^{20}$ The details of the calculations are described in Appendix B. With this model, values for $T_{c}$ can be calculated for each order parameter configuration, since these are the eigenvalues of the matrix equations. The number of $T_{c}$ values is obviously given by $N_{\text {bi }}$. They will be called $T^{(k)}$, where $T^{(0)}$ denotes the configuration with zero nodes (the first symmetric solution), and $T^{\left(N_{\mathrm{bi}}-1\right)}$ the configuration with $N_{\mathrm{bi}}-1$ nodes.

\section{B. An example: The five-bilayer system}

To demonstrate the applicability of our approach, and in order to make the main features of the studied multilayers more transparent, we start with the examination of the simplest generic $\mathrm{F} /[5(\mathrm{~S} / \mathrm{F})]$ structure, an asymmetric multilayer consisting of five bilayers and a closing $F$ layer. It contains

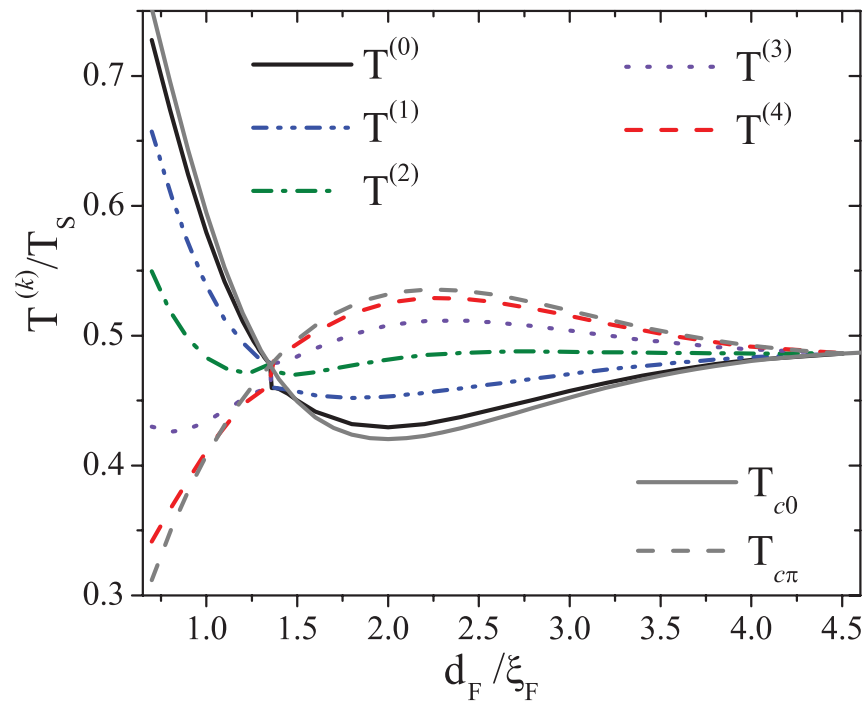

FIG. 6. (Color online) Eigenvalues of the critical temperature $T^{(k)}$ of the $\mathrm{F} /[5(\mathrm{~S} / \mathrm{F})]$ structure as a function of $\mathrm{F}$ layer thickness $d_{F}$, calculated for $d_{S}=4.67 \xi_{S}$. Also shown are the solutions for the infinite multilayer $T_{c 0}$ and $T_{c \pi}$.

the smallest amount of blocks necessary to demonstrate the effects we have observed experimentally in more complex systems. We need materials constants for the calculation, and we have chosen them close to ones of $\mathrm{Nb} / \mathrm{PdNi}$ bilayers ${ }^{9}$; namely, $\rho_{S} / \rho_{F}=0.29, \gamma_{b} \xi_{F}^{*} / \xi_{F}=0.28$, and $\xi_{F}=0.5 \xi_{S}$, where $\xi_{F} \equiv \sqrt{D_{F} / E_{\mathrm{ex}}}$ is the characteristic decay length of the order parameter. We also take the thickness of the $S$ layer large enough $\left(d_{S}=4.7 \xi_{S}\right)$ in order to satisfy the conditions of the single mode approximation. ${ }^{21}$ Figure 6 shows the thickness dependence of eigenvalues $T^{(k)}\left(d_{F}\right)$ for five-bilayer system. Also shown are the results for the infinite multilayer calculation, with the symmetric solution $T_{c 0}$ (solid gray line) and the antisymmetric solution $T_{c \pi}$ (dashed gray line). The eigenvalues $T^{(k)}$ are numbered according to the number of zeros of appropriate eigenvector functions $\Phi^{(k)}(z)$ (see Appendix B for details).

It is clearly seen that there is an intersection of all the curves $T^{(k)}\left(d_{F}\right)(k=0, \ldots, 4)$, which occurs in a narrow regions in the vicinity of $0-\pi$ crossover $d_{F}^{*} \approx 1.35 \xi_{F}$. In the interval $d_{F}<d_{F}^{*}$ the hierarchy in $T^{(k)}$ is $T^{(k+1)}<T^{(k)}$, while for $d_{F}>d_{F}^{*}$ this is changed to the opposite $T^{(k+1)}>T^{(k)}$. For $d_{F}<d_{F}^{*}$, the zero-node state occurs, as described by eigenvector function $\Phi^{(0)}(z)$ shown in Fig. 7(a). Above $d_{F}^{*}$ the system goes into the four-node state, which is basically a $\pi$ state, and described by the eigenvector function $\Phi^{(4)}(z)$ [Fig. 7(b)].

Now let us focus on the immediate proximity to the point of $0-\pi$ crossover and understand the evolution the order parameter is going through. Figure 8 shows the detailed variation of $T^{(k)}$ around the crossing point at $d_{F}^{*}$. Looking closely, all curves evolve in a nonmonotonous fashion. The solutions $T^{(0,1)}$ show a jump down, the solutions $T^{(3,4)}$ show a jump up, and $T^{(2)}$ shows a kink at $d_{F}^{*}$. However, there is continuity in the variation of the eigenvalues in the sense that at the degeneracy point the variation in $T^{(0)}$ is continued in $T^{(2)}$, as is $T^{(1)}$ continued in $T^{(3)}, T^{(2)}$ in $T^{(4)}, T^{(3)}$ in 


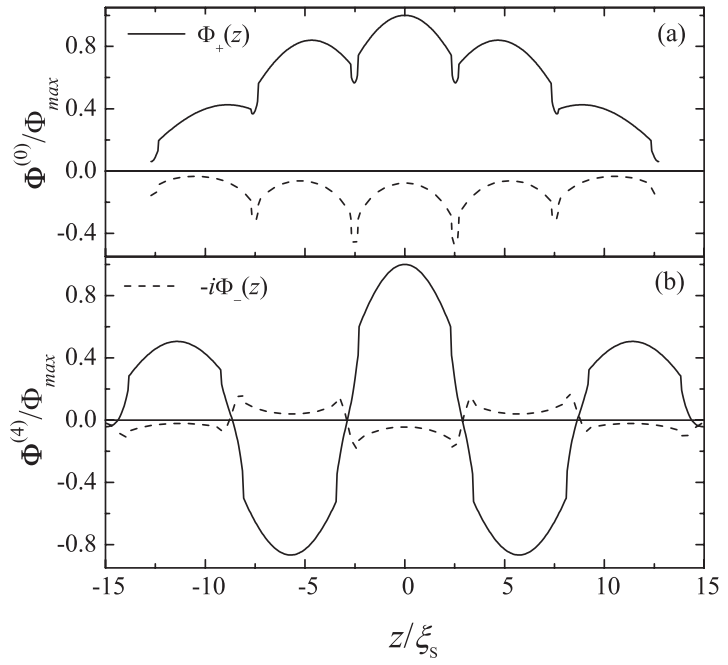

FIG. 7. Real $\Phi_{+}(z)$ and imaginary $\left[-i \Phi_{-}(z)\right]$ parts of eigenvector functions (a) $\Phi^{(0)}(z)$ and (b) $\Phi^{(4)}(z)$ calculated for $d_{F}=0.7 \xi_{F} \approx$ $0.52 d_{F}^{*}$ and $d_{F}=2.2 \xi_{F} \approx 1.63 d_{F}^{*}$, respectively.

$T^{(1)}$, and $T^{(4)}$ in $T^{(0)}$. The evolution of the eigenfunctions reflect this change in the sense that, in the vicinity of the degeneracy point, they start to take on the shape of the continuation.

For instance, the eigenfunction $\Phi^{(0)}(z)$ transform from the shape at $d_{F}=0.7 \xi_{F}$ given in Fig. 7(a) to the one at $d_{F}=1.33 \xi_{F}$ (very close to the degeneracy point) shown in Fig. 9(a). The similarity to a two-node function is becoming apparent, which is the way in which the eigenvalue continues. Similarly, the eigenvalue with the proper function $\Phi^{(4)}(z)$ is a continuation of the one with $\Phi^{(2)}(z)$ and at $d_{F}=1.37 \xi_{F}$ (just beyond the degeneracy point) $\Phi^{(4)}(z)$ still shows strong resemblance to a two-node function as can be seen in Fig. 9(c).

Figure 9 is also meant to make another point; namely, how the ground state of the system, casu quo the highest eigenvalue (which is $T_{c}$ ) evolves around $d_{F}^{*}$. Figures 9(a)-9(c) present those eigenfunctions which in turn determine $T_{c}$ when increasing $d_{F}$ from $1.33 \xi_{F}$ up to $1.37 \xi_{F}$; namely, $\Phi^{(0)}(z)$, $\Phi^{(1)}(z)$, and $\Phi^{(4)}(z)$. An interesting feature of the crossover

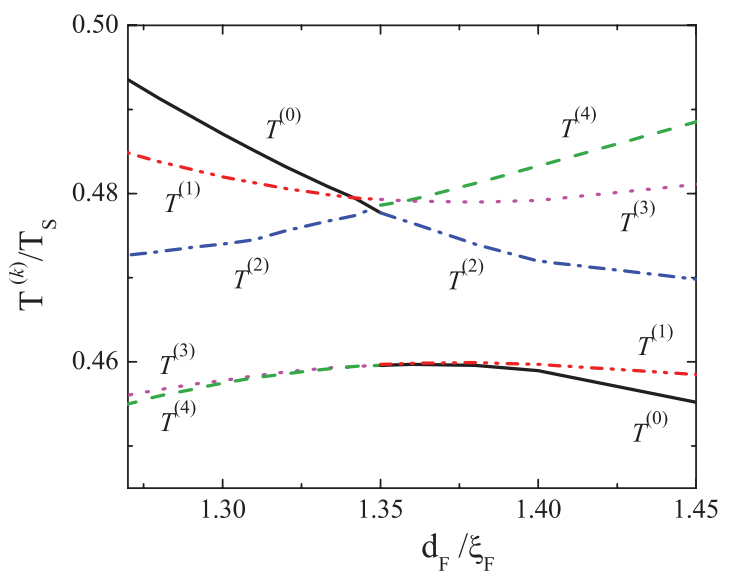

FIG. 8. (Color online) Eigenvalues of critical temperature $T^{(k)}$ of $\mathrm{F} /[5(\mathrm{~S} / \mathrm{F})]$ structure as a function of $\mathrm{F}$ layer thickness in immediate proximity to the point of $0-\pi$ crossover (see Fig. 6).

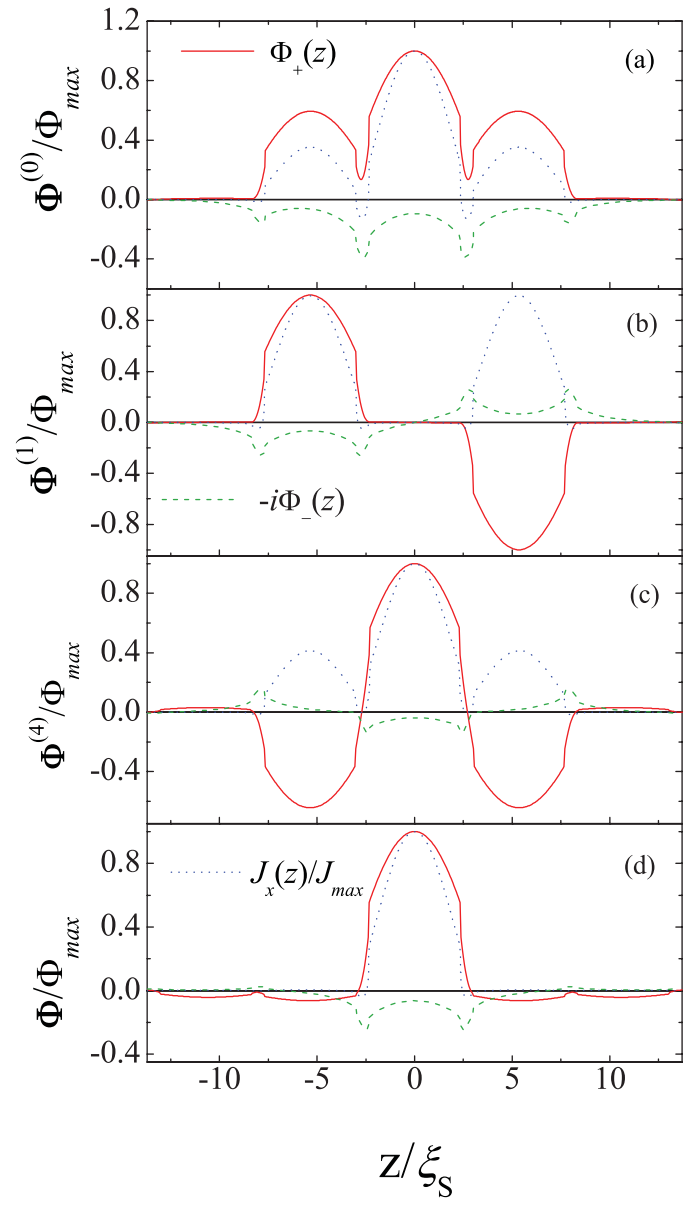

FIG. 9. (Color online) Real $\Phi_{+}(z)$ and imaginary $\left[-i \Phi_{-}(z)\right]$ parts of eigenvector functions and supercurrent density $J_{x}(z)$ calculated for the $\mathrm{F} /[5(\mathrm{~S} / \mathrm{F})]$ multilayer for (a) $d_{F}=1.33 \xi_{F}$, (b) and (d) $d_{F}=$ $1.35 \xi_{F}$, and (c) $d_{F}=1.37 \xi_{F}$.

is that upon approaching $d_{F}^{*}$, superconductivity is practically suppressed in the outward layers, while exactly at the crossover it disappears in the central layer. However, this solution is only marginally lower in energy than the solution emerging from $\Phi^{(0,2,4)}(z)$ given in Fig. 9(d). Another interesting feature is the behavior of the spatial distribution of the supercurrent density $J_{x}(z)$, which shows weak countercurrents flowing along the central ferromagnetic layers [see Fig. 9(a)]. It means that a measurement current will actually be accompanied by countercurrents.

\section{Engineering an order parameter}

From the above it will be clear that the system goes through strong evolutions in the neighborhood of $d_{F}^{*}$ (and more so than in the symmetric case), but also that for other thicknesses in a regular $\mathrm{F} /\left[N_{\mathrm{bi}}(\mathrm{S} / \mathrm{F})\right]$ only the eigenfunctions with eigenvalues $T^{\left(0, N_{\mathrm{bi}}-1\right)}$ occur, and that the other eigenfunctions remain hidden (see Fig. 6). On the other hand, there is a rather simple solution for engineering the occurrence of the other states, ${ }^{22}$ by slightly changing the thickness of even one layer. For example, increasing the thickness of a central F layer (for even $N_{\mathrm{bi}}$ ), or decreasing the thickness of a central $\mathrm{S}$ layer (for odd $N_{\mathrm{bi}}$ ), will force a node into that layer and the corresponding state 


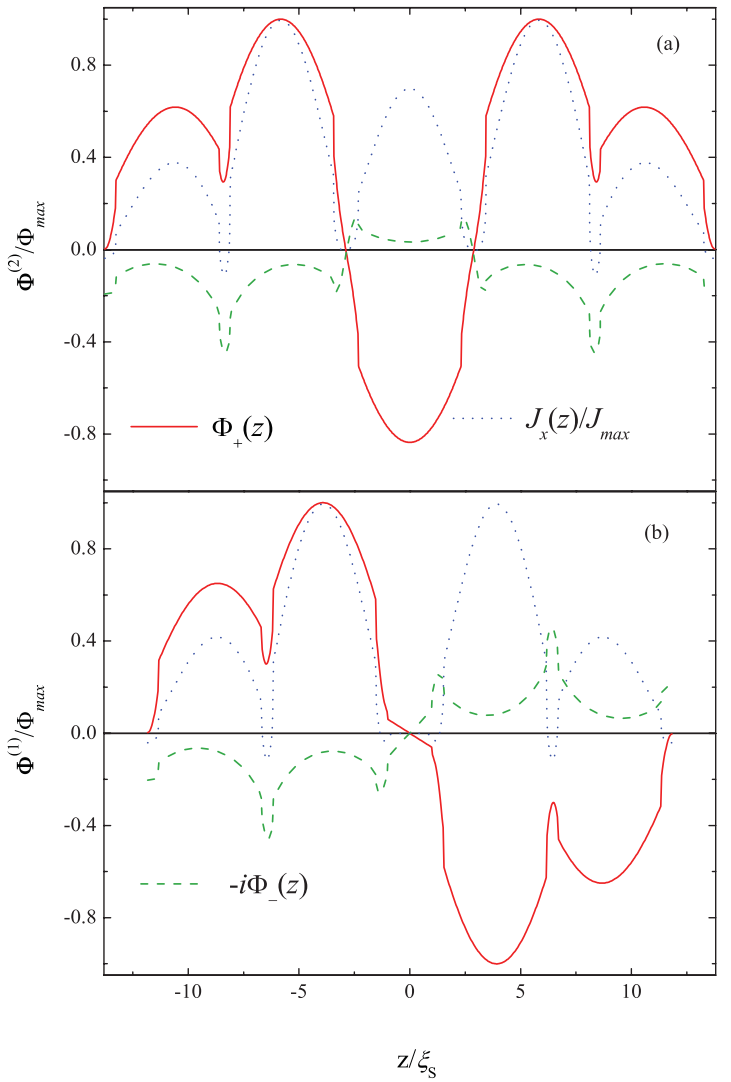

FIG. 10. (Color online) Eigenvector functions and supercurrent density $J_{x}(z)$ calculated for the $\mathrm{F} /[5(\mathrm{~S} / \mathrm{F})]$ multilayer with (a) enlarged thickness of the two central $F$ layers and (b) with reduced thickness of the central S layer.

determines the multilayer critical temperature. Our numerical calculations confirm this statement. Figure 10 gives the spatial distribution of the eigenfunctions calculated for two particular structures of the $\mathrm{F} /[5(\mathrm{~S} / \mathrm{F})]$ model system, which has a central S layer. In the first case [Fig. 10(a)], the thicknesses of the F layers directly contacting the central S layer are $d_{F}=2.2 \xi_{F}$, while the other $\mathrm{F}$ layers have $d_{F}=\xi_{F}$. In the second case [Fig. 10(b)], the central S layer thickness $d_{S}=2 \xi_{S}$, while the other $\mathrm{S}$ layers have $d_{S}=4.67 \xi_{S}$. In the first case, the ground state becomes $\Phi^{(2)}(z)$, while in the second it is $\Phi^{(1)}(z)$. It is important to note that also in these configurations (as also shown in Fig. 10) the application of a measuring current into the structure will be accompanied by generation of countercurrents.

Obviously, in the presence of structural inhomogeneities in any true structure consisting of $N_{\mathrm{bi}}(\mathrm{F} / \mathrm{S})$ bilayers, it will be energetically favorable for the countercurrents to be closed by forming current loops having the smallest characteristic dimension. It means that such a multilayer is unstable against the formation of clusters of current loops in the transition from the normal to the superconducting state. Moreover, for the set of parameters under which a multilayer is close to the 0 to $\pi$ transition such current loops (stretching over varying numbers of bilayer blocks) should, in the transition, leave fingerprints of the otherwise hidden eigenvalues $T^{(k)}$ of smaller blocks within the full structure. This we believe to be is what we essentially observe.

\section{ANALYSIS AND DISCUSSION}

We use the theoretical model developed above to study quantitatively the variation of $\Delta T_{c}$ as found in the series $\mathrm{MB} n$ as well as in the stepped structure in MA9. The parameters to be chosen and varied are the usual ones for proximity effect problems. Fixed are $T_{c S}=9.2 \mathrm{~K}$ (the bulk value for $\mathrm{Nb}$ ) and $\omega_{D}=275 \mathrm{~K}$. Fitting parameters then in principle are the proximity parameter $\gamma=\rho_{S} \xi_{S} /\left(\rho_{F} \xi_{F}\right)$ and the transparency parameter $t_{b}$. However, the fitting strategy is not straightforward for the multilayers, since it is not simply $T_{c}$ which is to be fitted. We therefore use a slightly different approach, and determine the necessary parameters using the basic multilayer building blocks MS1 and MS2, which do have a well-determined $T_{c}$ and a transition width of $50 \mathrm{mK}$. We optimize the following parameters around the values found in Ref. 9: $\xi_{S}=5.6 \mathrm{~nm}(5.6 \mathrm{~nm}), \xi_{F}^{*}=5.0 \mathrm{~nm}(6.2 \mathrm{~nm})$, $p=\rho_{S} / \rho_{F}=0.15(0.26)$, and $\gamma_{b}=0.22(0.13)$, where the values in brackets are the ones used in Ref. 9. The current parameter set is quite close to the previous one, which gives confidence in the procedure. We then use these parameters to calculate the sets $T_{n}$ for the series $\mathrm{MB} n$. The values for the $T^{(0)} \approx T_{c 0}$ (no nodes) and $T^{(8)} \approx T_{c \pi}$ (largest number of nodes) are given in Fig. 4.

The first striking conclusion is that the transition widths for large $N_{\mathrm{bi}}$ are roughly given by the spread of $T^{(k)}$. In particular, the upper limit is very well reproduced by the zero-node solutions (open circles in Fig. 4). The lower limit (open squares) is the maximum nodal state. The critical temperature for the lower limit is always less than the measured values, for reasons to be discussed below. Also clear is that below $N_{\mathrm{bi}}=5$, the transition singles out only one mode. Before discussing the behavior of these samples in more detail, we turn to the multistep transition of sample MA9, shown in Fig. 3 and enlarged in Fig. 11. The typical behavior of our

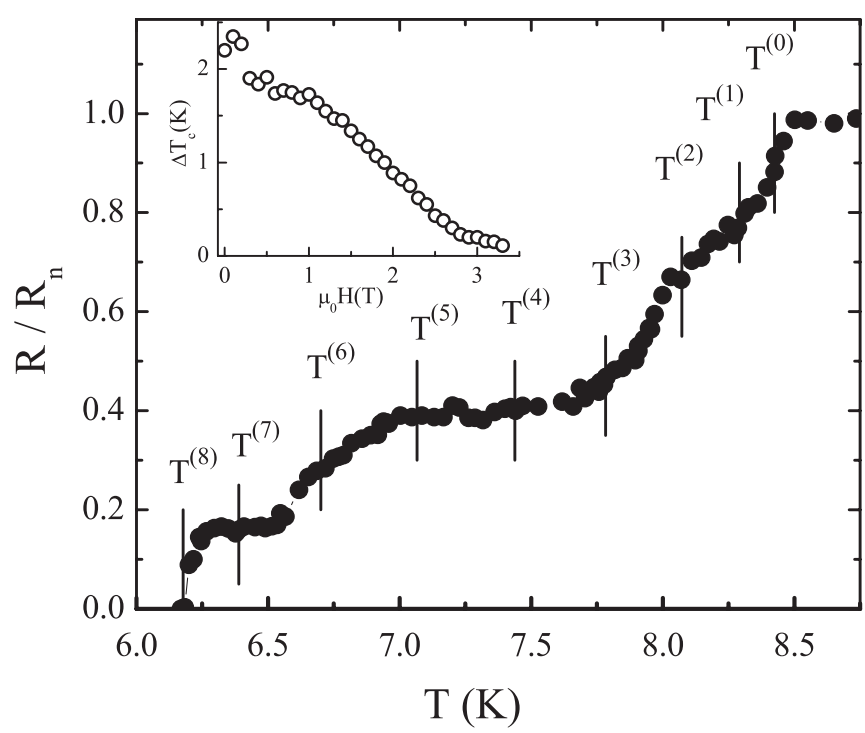

FIG. 11. Normalized resistive transition for sample MA9. Vertical lines mark the positions of all $T^{(k)}$ values as calculated using the materials parameters given in the main text. Inset: the width of the resistive transitions $\Delta T_{c}$ given in Fig. 5(b) as function of applied in-plane magnetic field. 
multilayer samples is a transition with two or three broad steps, but here a number of visible steps is larger. For this sample we examined whether all nine $T^{(k)}$ s can be reasonably fitted in the transition. This turns out to work surprisingly well. Using $\xi_{S}=5.2 \mathrm{~nm}(5.6 \mathrm{~nm}), \xi_{F}^{*}=5.2 \mathrm{~nm}(5.0 \mathrm{~nm})$, $p=\rho_{S} / \rho_{F}=0.10(0.15)$, and $\gamma_{b}=0.16$ (0.22) (with the values in parentheses now the ones used in fitting the $\mathrm{MB} n$ series), we find $T^{(k)}$ values as plotted in Fig. 11. We do not claim that we literally observe these $T^{(k)} \mathrm{s}$. The point is rather that this sample spans the full transition width of about $2.5 \mathrm{~K}$ allowed by its physical parameters, which means that for MA9 the transition to superconductivity starts with the 0-node state and ends with the maximum nodal configuration.

The unequivocal message from the experiments is that our large- $N_{\mathrm{bi}}$ multilayers have transition widths which are connected to the different possible order parameter configurations, starting with the 0-node symmetric one. Still, it is not as if the system sequentially samples them; in that case the highest of the possible $T_{c} \mathrm{~s}$ in the system would determine the transition temperature. The picture is a bit more subtle, and for the discussion we refer to a recent study we performed on the transition width in simple $\mathrm{S} / \mathrm{F} / \mathrm{S}$ trilayers made with $\mathrm{Nb}$ and $\mathrm{Cu}_{41} \mathrm{Ni}_{59}$, where $d_{F}$ was varied through the $0-\pi$ transition region. ${ }^{10}$ In that region, similar broadenings were found as in the multilayers under discussion, although the values of $\Delta T_{c}$ were significantly less, not more than $0.5 \mathrm{~K}$. For the trilayers we proposed a model in which, under the influence of small variations in thickness, interface roughness, and exchange energies, the system in the transition actually has to be viewed as a network of S-N-S and S-F-S junctions. At the onset of superconductivity, superconducting islands start to form in the $\mathrm{S}$ layers, separated by still normal regions in the same layer, while the islands are also connected to islands in other layers through the weak $\mathrm{F}$ material. Local loops between two $\mathrm{S}$ layers (1 and 2) may now emerge of type $\mathrm{S}_{1}-\mathrm{N}_{1}-\mathrm{S}_{1}-\mathrm{F}-\mathrm{S}_{2}-\mathrm{N}_{2}-\mathrm{S}_{2}-\mathrm{F}-\mathrm{S}_{1}$, containing two $\mathrm{S}-\mathrm{F}-\mathrm{S}$ junctions. If one of these goes into a $\pi$ state, the loop can maintain a circulating Josephson current which works against the growth of the $\mathrm{S}$ islands and broadens the transition. Of course, once the $\mathrm{S}$ islands coalesce, the circulating currents disappear and the system is in a well-defined state with respect to the phases.

Obviously a similar mechanism should be at work in our multilayers, where the F-layer thickness is in the region of allowing $\pi$ states. But now the multilayer allows a larger variety of networks to be formed on the basis of order parameter configurations with numbers of nodes up to $N_{\mathrm{bi}}-1$. The picture which then emerges is that the first networks which occur below the onset of superconductivity are like 0 -node configurations. Going down in the transition, loops between two adjacent $\mathrm{S}$ layers survive longest, which is like the full-nodal configuration, although this configuration is not necessarily reached. For that, the structure should have the correct periodical structure, and if that condition is not fulfilled, the system separates in different subblocks having a smaller number of nodes and a higher $T_{c}$. In our case the F-layer thickness is not optimal, and we usually find $T_{c}$ for the system above that was predicted by the full nodal solution. It is worth noting that a similar model of Josephson networks with 0 and $\pi$ contacts was considered almost 20 years ago in connection with the paramagnetic Meissner effect ("Wohlleben effect")
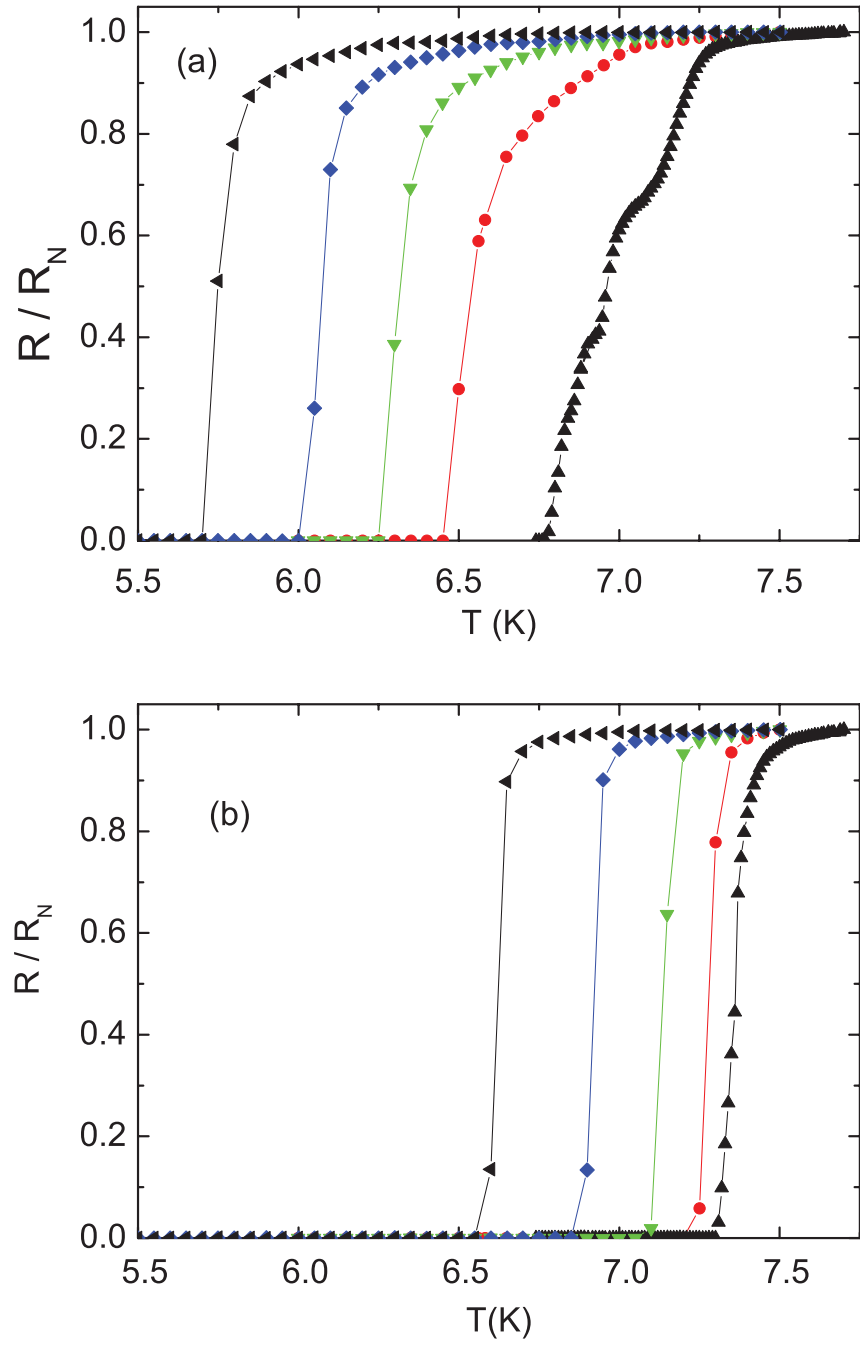

FIG. 12. (Color online) Resistance $R$ vs temperature $T$ for two different sets of multilayers consisting of building blocks $\mathrm{Cu}_{0.41} \mathrm{Ni}_{0.59}(3 \mathrm{~nm}) / \mathrm{Nb}(30 \mathrm{~nm})$, and in different applied magnetic fields; from right to left: $(0,0.25,0.5,0.75,1) \mathrm{T}$. (a) is an asymmetric set of type $\mathrm{F} /[\mathrm{g}(\mathrm{S} / \mathrm{F})]$; (b) is a symmetric set where the outer $\mathrm{F}$ layers have half the thickness of the inner ones.

in high- $T_{c}$ ceramics. $^{23-26}$ The main difference with our work is that the finite $\mathrm{S} / \mathrm{F}$ multilayer can spontaneously divide into fragments with different $T_{c} \mathrm{~s}$.

The measurements of the field dependence of the transition performed on sample MA9 [see Fig. 5(a)] are in line with this picture. The variation of the widths of the transition as a function of applied $H_{a}$ are given in the inset of Fig. 11 . They show that for decreasing temperature, $\Delta T_{c}$ continuously decreases from a value around $2 \mathrm{~K}$ at $0 \mathrm{~T}$ to $0.1 \mathrm{~K}$ at $3 \mathrm{~T}$. This again illustrates that the broadening is not simply due to static sample inhomogeneities. Similar to the trilayer case, we ascribe this to a breaking up of the Josephson networks in higher magnetic fields, resulting in sharper transitions. The experiment with sample MA9-35 adheres to the same picture, increasing the central S-layer thickness also results in a sharper transition (see Fig. 3): coalescence of superconducting islands in the thicker layer will occur more quickly than in the other layers, shorting still existing networks in the multilayer. 
Finally, to show the generality of our results, we prepared a multilayer set on the basis of the weak ferromagnet $\mathrm{Cu}_{0.41} \mathrm{Ni}_{0.59}$ (called $\mathrm{CuNi}$ ), also used in earlier experiments. ${ }^{27}$ The asymmetric sample consisted of nine blocks $\mathrm{CuNi}(3 \mathrm{~nm}) /$ $\mathrm{Nb}(30 \mathrm{~nm})$, with a closing layer of $\mathrm{CuNi}(3 \mathrm{~nm})$ covered by $\mathrm{Nb}(2 \mathrm{~nm})$ to prevent oxidation. The symmetric sample had outer layers of $1.5 \mathrm{~nm} \mathrm{CuNi}$. The results in Fig. 12 sketch exactly the same picture as for the multilayers with PdNi. The thickness of the $\mathrm{CuNi}$ layer is close to the transition to the $\pi$ state, and the asymmetric sample has a transition width of about $0.5 \mathrm{~K}$, which sharpens to $0.2 \mathrm{~K}$ in a field of $1 \mathrm{~T}$. The symmetric sample shows transition widths of less then $0.1 \mathrm{~K}$. The smaller widths in the asymmetric case compared to the PdNi samples is probably due to the somewhat larger thickness of the $\mathrm{Nb}$ layers, which result in a $T_{c}$ around $7.5 \mathrm{~K}$. In conclusion, we have worked out the concept of different possible order parameter configurations in S/F multilayers. We have used this in analyzing the increasing transition width in $\mathrm{S} / \mathrm{F}$ multilayers in terms of simultaneously emerging superconducting islands and Josephson junction networks, and we have shown that this width can actually be quantitatively predicted on the basis of the possible $T_{c} \mathrm{~s}$ of the system as given by the different configurations. We also showed how the model we developed allows us to engineer different order parameter configurations. It would seem that this property of S/F multilayers could lead to novel devices if they can be brought to switch between different configurations, in particular between zero, one, and two nodes. This will be an area of future research.

\section{ACKNOWLEDGMENTS}

The work has been partially supported by RFBR-BFBR Grants No. 12-02-90010 (M.Yu.K.) and No. F10R-063 (V.N.K. and S.L.P.), by the Italian MIUR-PRIN 2007 project "Proprietà di trasporto elettrico dc e ac di strutture ibride stratificate superconduttore/ferromagnete realizzate con materiali tradizionali"(C.C. and C.A.), by the CNR within the CNR Short Term Mobility Program (C.C.), and by the "Stichting voor Fundamenteel Onderzoek der Materie (FOM)" (J.A.). J.M. v.d Knaap is acknowledged for the preparation of the $\mathrm{Nb} / \mathrm{CuNi}$ multilayers. The research leading to these results and the final preparation of the manuscript has received funding from FP7/2007-2013 under Grant No. 264098-MAMA. Support from the ESF-programme "Nanoscience and Engineering in Superconductivity" (NES) is also acknowledged.

\section{APPENDIX A: $d_{F}$ AT THE 0- $\pi$ TRANSITION}

Generally, many factors (among which, e.g., the transparency of the interfaces and the strength of the magnetic scattering) determine the thickness $d_{F, \text { cr }}$ at which an $S / F$ multilayer crosses over from the 0 state to the $\pi$ state, and it is not possible to give a simple estimate in terms of what is actually a complex coherence length $\xi_{F 1}+i \xi_{F 2}$, where $\xi_{F 1}$ sets the decay length and $\xi_{F 2}$ sets the oscillation length of the order parameter. We neglect magnetic scattering, as we did above, so that both lengths are given by $\xi_{F}$ (and the oscillation wavelength by $\lambda_{F}=2 \pi \xi_{F}$ ). Under that assumption, an estimate can be given for some simple cases.
In the case of an S/F bilayer, Fominov et al. ${ }^{21}$ find for a fully transparent interface that $4 d_{F, \mathrm{cr}} / \lambda_{F} \approx 0.7$, which means the ratio $d_{F, \mathrm{cr}} / \xi_{F}=0.35 \pi=1.1$. It was argued in Ref. 21 that this result can be qualitatively understood in terms of a simple interference picture which yields $d_{F, \mathrm{cr}} / \xi_{F}=(\pi / 4) \approx 1.55$, which still overestimates the actual value by about $40 \%$. If the interface is less than fully transparent, the ratio goes down, but also the minimum in $T_{c}\left(d_{F}\right)$ disappears. For the case of the infinite $\mathrm{S} / \mathrm{F}$ multilayer with fully transparent interfaces, the calculations by Radovic et al. yielded $d_{F, \text { cr }} / \xi_{F} \approx 1,{ }^{8}$ while Buzdin $^{1}$ finds the first $0-\pi$ transition to occur at $d_{F, \text { cr }} / \xi_{F} \approx$ 1.18. Again, when the interface transparency decreases, the ratio goes down. Note that for the case of the asymmetric five-layer system above, we find $d_{F, \mathrm{cr}} / \xi_{F} \approx 1.35$, still quite close to the (symmetric) infinite multilayer case.

Next to the minimum in $T_{c}$, also the minimum in the critical current $I_{c, \min }$, can be used to find the crossover. Near $T_{c}$ this minimum is found at the same $d_{F, \mathrm{cr}}$ as for the $T_{c}$ minimum; namely at $1.18 \xi_{F},{ }^{1}$ and at somewhat lower value for lower temperatures. Also here, the introduction of insulating (I) barriers, leading to SIFS or SIFIS configurations, can strongly lower the value of the crossover thickness. ${ }^{1,28}$ The bottom line is that in general, the crossover value depends on many parameters, with few simple rules.

Experimentally, values for $\xi_{F}$ in $\mathrm{Pd}_{1-x} \mathrm{Ni}_{x}$ are as follows. For $x=0.12$, Khaire et al. find an oscillation length $\xi_{F 2}$ of $4.4 \mathrm{~nm}$ from the critical current in $\mathrm{S} / \mathrm{F} / \mathrm{S}$ junctions with relatively clean layers. Baladié and Buzdin find $\xi_{F}=3.0 \mathrm{~nm}$ by reanalyzing the data of Kontos et al. ${ }^{29,30}$ in the correct SIFS junction geometry. For $x=0.14$, Cirillo et al. found $\xi_{F}=3.4 \mathrm{~nm}$ from a $T_{c}$ analysis of S/F bilayers, ${ }^{31}$ and Matsuda et al. found $\xi_{F}=3.5 \mathrm{~nm}$, also from the $T_{c}$ of bilayers. ${ }^{32}$ For $x=0.19$, the value of the present samples, Cirillo et al. found a $T_{c}$ minimum around $3.0 \mathrm{~nm}$ in bilayers. Neglecting magnetic scattering, and noting that the interface transparency in these bilayers is rather high, this yielded a value of $\xi_{F}=$ $2.8 \mathrm{~nm}^{9}$; similarly, a $T_{c}$ study of SFS trilayers also yielded $\xi_{F}=2.8 \mathrm{~nm} .{ }^{33}$ Note the more or less monotonic decrease of $\xi_{F}$ with increasing Ni concentration. For a symmetric infinite multilayer with highly transparent interfaces, the crossover can therefore be expected somewhat below the full transparency value of $1.2 \xi_{F}=3.4 \mathrm{~nm}$. We estimate the transparency effect to yield $3.1 \mathrm{~nm}$. The thickness used in the experiment is a little bit lower than that, but this does not appear to be critical.

\section{APPENDIX B: THEORETICAL DETAILS}

It is convenient to write the solution of boundary problem (1)-(5) in the matrix form ${ }^{20}$

$$
\mathbf{Y}(z)=\hat{\mathbf{R}}(z) \mathbf{Y}(-L / 2)
$$

where $\mathbf{Y}(z)$ is the direct sum $\Phi_{+}(z) \oplus \Phi_{+}^{\prime}(z) \oplus \Phi_{-}(z) \oplus$ $\Phi_{-}^{\prime}(z)$, where $\Phi_{ \pm}(z)=\left(\Phi_{ \pm, 0} \Phi_{ \pm, 1} \cdots \Phi_{ \pm, n_{D}+1}\right)^{\text {tr }}$ are $\left(n_{D}+\right.$ 1)-dimensional vector functions (by the symbol tr we denote here the transposition operation) related to $F_{n}$ by $\Phi_{ \pm, n}=$ $\left(F_{n} \pm F_{-n-1}\right) / 2$, and $\hat{\mathbf{R}}(z)$ is the matrizant of the system (1)-(4). [The matrizant is the matrix of fundamental system solutions, which is satisfied by the condition $\hat{\mathbf{R}}(-L / 2)=\hat{\mathbf{1}}, \hat{\mathbf{1}}$ 
is the unit matrix.] Substitution of (B1) into (5) leads to the following system of linear uniform algebraic equations:

$$
\hat{\mathbf{R}}_{24,13}(L / 2) \Phi(-L / 2)=\mathbf{0} .
$$

Here $\Phi=\Phi_{+} \oplus \Phi_{-}$is a column vector, while

$$
\hat{\mathbf{R}}_{24,13}=\left(\begin{array}{c}
\hat{\mathbf{R}}_{2,1} \hat{\mathbf{R}}_{2,3} \\
\hat{\mathbf{R}}_{4,1} \hat{\mathbf{R}}_{4,3}
\end{array}\right),
$$

where $\hat{\mathbf{R}}_{\alpha, \beta}(\alpha, \beta=1,2,3,4)$ are $\left(n_{D}+1\right) \times\left(n_{D}+1\right)$ matrix blocks of matrix $\hat{\mathbf{R}}$.

The condition of existence of nontrivial solutions of the system (B2) gives the characteristic equation in the form

$$
\operatorname{det}\left[\hat{\mathbf{R}}_{24,13}(L / 2)\right]=0 .
$$

The matrizant $\hat{\mathbf{R}}$ in Eq. (B4) can be found in explicit form and is expressed in terms of the product of the matrizants of $\mathrm{S}-(\hat{\mathbf{S}})$ and F-( $\hat{\mathbf{M}})$ layers and the matrices $\hat{\Gamma}_{S F}, \hat{\Gamma}_{F S}$ determined by the matching conditions (3), (4). In particular for the structures $\mathrm{F} / N_{b l}(\mathrm{~S} / \mathrm{F})$ the matrizant, which is connected vectors $\mathbf{Y}(-L / 2)$ and $\mathbf{Y}(L / 2)$, has the form

$$
\hat{\mathbf{R}}(L / 2)=\hat{\mathbf{M}}\left(d_{F}\right)\left[\hat{\Gamma}_{F S} \hat{\mathbf{S}}\left(d_{S}\right) \hat{\Gamma}_{S F} \hat{\mathbf{M}}\left(d_{F}\right)\right]^{N_{b l}} .
$$

Matrices $\hat{\mathbf{S}}$, $\hat{\mathbf{M}}$, and $\hat{\Gamma}_{S F(F S)}$ can be written as

$$
\hat{\mathbf{S}}(z)=\left(\begin{array}{lc}
\hat{\mathcal{C}} \hat{\mathbf{S}}_{+}(z) \hat{\mathcal{C}}^{\mathrm{tr}} & \hat{\mathbf{0}} \\
\hat{\mathbf{0}} & \hat{\mathbf{S}}_{-}(z)
\end{array}\right)
$$

and matrices $\hat{\mathbf{S}}_{+}(z)$ and $\hat{\mathbf{S}}_{-}(z)$ in (B6) are given by

$$
\hat{\mathbf{S}}_{ \pm}(z)=\left(\begin{array}{lc}
\operatorname{diag}\left[\cosh \left(k_{n}^{ \pm} z\right)\right] & \operatorname{diag}\left[\left(k_{n}^{ \pm}\right)^{-1} \sinh \left(k_{n}^{ \pm} z\right)\right] \\
\operatorname{diag}\left[k_{n}^{ \pm} \sinh \left(k_{n}^{ \pm} z\right)\right] & \operatorname{diag}\left[\cosh \left(k_{n}^{ \pm} z\right)\right]
\end{array}\right) .
$$

Here $\operatorname{diag}\left[a_{n}\right]$ is diagonal matrix with the main diagonal elements $a_{0}, a_{1}, \ldots, a_{n_{D}} ; k_{n}^{+}=\xi_{S}^{-1} \sqrt{-2 T \mu_{n} / T_{c S}}, k_{n}^{-}=$ $\xi_{S}^{-1} \sqrt{(2 n+1) T / T_{c S}}$, where $\mu_{n} \equiv \mu_{n}(T)$ are the roots of the equation

$$
\begin{gathered}
\psi\left(\frac{\omega_{D}}{2 \pi T}+\mu+1\right)-\psi\left(\frac{1}{2}+\mu\right) \\
\quad=\psi\left(\frac{\omega_{D}}{2 \pi T_{c S}}+1\right)-\psi\left(\frac{1}{2}\right),
\end{gathered}
$$

where $\psi(t)$ is the digamma function.

The matrix $\hat{\mathcal{C}}$ in (B6) has the form

$$
\hat{\mathcal{C}}=\left(\begin{array}{ll}
\hat{\mathbf{C}} & \hat{\mathbf{0}} \\
\hat{\mathbf{0}} & \hat{\mathbf{C}}
\end{array}\right)
$$

where $\hat{\mathbf{C}}$ is an orthogonal matrix, which is intended by vectors

$$
c_{n m}=\frac{2 s_{m}}{2 n+1+2 \mu_{m}},
$$

containing the normalized coefficients

$$
s_{m}=\left[\sum_{l=0}^{n_{D}} \frac{4}{\left(2 l+1+2 \mu_{m}\right)^{2}}\right]^{-1 / 2} .
$$

For matrizants of the F layer it is easy to get

$$
\hat{\mathbf{M}}(z)=\left(\begin{array}{lc}
\operatorname{Re}[\hat{\mathbf{m}}(z)] & \iota \operatorname{Im}[\hat{\mathbf{m}}(z)] \\
l \operatorname{Im}[\hat{\mathbf{m}}(z)] & \operatorname{Re}[\hat{\mathbf{m}}(z)]
\end{array}\right),
$$

where

$$
\hat{\mathbf{m}}(z)=\left(\begin{array}{lc}
\operatorname{diag}\left[\cosh \left(\kappa_{n} z\right)\right] & \operatorname{diag}\left[\left(\kappa_{n}\right)^{-1} \sinh \left(\kappa_{n} z\right)\right] \\
\operatorname{diag}\left[\kappa_{n} \sinh \left(\kappa_{n} z\right)\right] & \operatorname{diag}\left[\cosh \left(\kappa_{n} z\right)\right]
\end{array}\right),
$$

and the characteristic lengths $\kappa_{n}$ are given by the following formula:

$$
\kappa_{n}=\frac{1}{\xi_{F}^{*}} \sqrt{\frac{\imath E_{\mathrm{ex}}+\omega_{n}}{\pi T_{c S}}} .
$$

Finally, from (3) and (4) it follows:

$$
\hat{\Gamma}_{F S(S F)}=\left(\begin{array}{lc}
\hat{\mathbf{P}}_{F S(S F)} & \hat{\mathbf{0}} \\
\hat{\mathbf{0}} & \hat{\mathbf{P}}_{F S(S F)}
\end{array}\right),
$$

where

$$
\hat{\mathbf{P}}_{F S}=\left(\begin{array}{cc}
\hat{\mathbf{1}} & \gamma_{b} \xi_{F}^{*} p^{-1} \hat{\mathbf{1}} \\
\hat{\mathbf{0}} & p^{-1} \hat{\mathbf{1}}
\end{array}\right), \quad \hat{\mathbf{P}}_{S F}=\left(\begin{array}{cc}
\hat{\mathbf{1}} & \gamma_{b} \xi_{F}^{*} \hat{\mathbf{1}} \\
\hat{\mathbf{0}} & p \hat{\mathbf{1}}=
\end{array}\right)
$$

In (B15) the parameter $p$ is the ratio of normal resistivities $\rho_{S} / \rho_{F}$, and the unit and zero matrices have $\left(n_{D}+1\right)$ dimensionality.

Thereby, the equations (B1)-(B15) determine the full solution of the boundary problem (1)-(5). From the characteristic equation (B4) and from Eq. (B2) one can get the $T^{(k)}$ eigenvalue set and the corresponding eigenvectors $\Phi^{(k)}(-L / 2)$, respectively. After that, making use of (B1), it is possible to find the eigenvector functions $\Phi^{(k)}(z)$. The largest eigenvalue from the $T^{(k)}$ eigenvalue set is the critical temperature $T_{c}$ of multilayer structure.

In an experiment, to get information about $T_{c}$, it is necessary to apply a measurement (transport) current $J_{x}$ in the direction parallel to the S-F boundary planes,for example, along the $O X$ axis. To find out the spatial distribution of this current in the direction perpendicular to the S-F interfaces direction $(O Z)$ we should take into account that this current is small. In first approximation we can then neglect the suppression of the $\Phi_{ \pm}$functions by this current and need not take into consideration the items in Eqs. (1) and (2) which are responsible for this depairing effect. We can further suppose that the existence of $J_{x}$ can be described by introducing factors $\exp (i k x)$ independent of $\omega$ in all $\Phi_{ \pm}$functions with the wave vector $k$ is proportional to the condensate superfluid velocity.

Substitution of that form of solution into an expression for the supercurrent density $1,34,35$

$$
\mathbf{J}=\frac{4 \pi T}{i e \rho(z)}\left(\Phi_{+}{ }^{* \mathrm{tr}} \nabla \Phi_{+}-\Phi_{-}{ }^{* \mathrm{tr}} \nabla \Phi_{-}-\text {c.c. }\right),
$$

results in $J_{x}(z)$ in the form

$$
J_{x}(z)=\frac{8 k \pi T}{e \rho(z)}\left(\Phi_{+}{ }^{* \mathrm{tr}} \Phi_{+}-\Phi_{-}{ }^{* \mathrm{tr}} \Phi_{-}\right) .
$$

Taking into account that for a given eigenvalue of the critical temperature $T^{(k)}$ there is the eigenvector function $\Phi^{(k)}(z)$, we 
can conclude that for every $T^{(k)}$ there is a well-defined spatial current distribution $J_{x}^{(k)}(z)$. It is necessary to mention that the existence of $\Phi_{-}(z)$ component in (B17) under certain conditions may lead to an alternation of sign of $J_{x}(z)$ with change of coordinate $z$, that is to generation of countercurrents in the S-F multilayer structures. This effect is the consequence of the existence of exchange interactions in $\mathrm{F}$ layers. In S-N multilayers $\Phi_{-}(z)$ is identically zero and these countercurrents do not exist.
${ }^{1}$ A. I. Buzdin, Rev. Mod. Phys. 77, 935 (2005).

${ }^{2}$ V. A. Oboznov, V. V. Bol'ginov, A. K. Feofanov, V. V. Ryazanov, and A. I. Buzdin, Phys. Rev. Lett. 96, 197003 (2006).

${ }^{3}$ A. Bauer, J. Bentner, M. Aprili, M. L. Della-Rocca, M. Reinwald, W. Wegscheider, and C. Strunk, Phys. Rev. Lett. 92, 217001 (2004).

${ }^{4}$ I. A. Garifullin, J. Magn. Magn. Mater. 240, 571 (2002).

${ }^{5}$ P. H. Barsic, O. T. Valls, and K. Halterman, Phys. Rev. B 75, 104502 (2007).

${ }^{6}$ S. Takahashi and M. Tachiki, Phys. Rev. B 33, 4620 (1986).

${ }^{7}$ A. I. Buzdin and M. Yu Kupriyanov, Pisma Zh. Eksp. Teor. Fiz. 52, 1089 (1990) [JETP Lett. 52, 487 (1990)].

${ }^{8}$ Z. Radović, M. Ledvij, L. Dobrosavljević-Grujić, A. I. Buzdin, and J. R. Clem, Phys. Rev. B 44, 759 (1991).

${ }^{9}$ C. Cirillo, A. Rusanov, C. Bell, and J. Aarts, Phys. Rev. B 75, 174510 (2007).

${ }^{10}$ S. L. Prischepa, C. Cirillo, C. Bell, V. N. Kushnir, J. Aarts, C. Attanasio, and M. Yu. Kupriyanov, Pis'ma Zh. Eksp. Teor. Fiz. 88, 431 (2008) [JETP Lett. 88, 375 (2008)].

${ }^{11}$ L. G. Parrat, Phys. Rev. 95, 359 (1954).

${ }^{12}$ L. Nevot and P. Croce, Rev. Phys. Appl. 15, 761 (1980).

${ }^{13}$ A. P. Payne and B. M. Clemens, Phys. Rev. B 47, 2289 (1993).

${ }^{14}$ C. L. Chien, J. S. Jiang, J. Q. Xiao, D. Davidovic, and D. H. Reich, J. Appl. Phys. 81, 5358 (1997).

${ }^{15}$ Y. Obi, M. Ikebe, and H. Fujishiro, Phys. Rev. Lett. 94, 057008 (2005).

${ }^{16}$ W.-C. Chiang, J. G. Lin, K. H. Hsu, D. S. Hussey, and D. V. Baxter, J. Magn. Magn. Mater. 304, E97 (2006).

${ }^{17}$ V. Shelukhin, A. Tsukernik, M. Karpovski, Y. Blum, K. B. Efetov, A. F. Volkov, T. Champel, M. Eschrig, T. Löfwander, G. Schön, and A. Palevski, Phys. Rev. B 73, 174506 (2006).
${ }^{18}$ K. D. Usadel, Phys. Rev. Lett. 25, 507 (1970).

${ }^{19}$ M. Y. Kuprianov and V. F. Lukichev, Zh. Eksp. Teor. Phys. 94, 139 (1988) [Sov. Phys. JETP 67, 1163 (1988)].

${ }^{20}$ V. N. Kushnir and M. Yu. Kupriyanov, Pis'ma v Zh. Eksp. Teor. Fiz. 93, 597 (2011).

${ }^{21}$ Ya. V. Fominov, N. M. Chtchelkatchev, and A. A. Golubov, Phys. Rev. B 66, 014507 (2002).

${ }^{22}$ A. Buzdin and A. E. Koshelev, Phys. Rev. B 67, 220504 (2003).

${ }^{23}$ W. Braunisch, N. Knauf, V. Kataev, S. Neuhausen, A. Grütz, A. Kock, B. Roden, D. Khomskii, and D. Wohlleben, Phys. Rev. Lett. 68, 1908 (1992).

${ }^{24}$ F. V. Kusmartsev, Phys. Rev. Lett. 69, 2268 (1992).

${ }^{25}$ S. V. Panyukov and A. D. Zaikin, Physica B 203, 527 (1994).

${ }^{26}$ D. Khomskii, J. Low Temp. Phys. 95, 205 (1994).

${ }^{27}$ M. Flokstra and J. Aarts, Phys. Rev. B 80, 144513 (2009).

${ }^{28}$ A. S. Vasenko, A. A. Golubov, M. Yu. Kupriyanov, and M. Weides, Phys. Rev. B 77, 134507 (2008).

${ }^{29}$ A. Buzdin and I. Baladié, Phys. Rev. B 67, 184519 (2003).

${ }^{30}$ T. Kontos, M. Aprili, J. Lesueur, F. Genet, B. Stephanidis, and R. Boursier, Phys. Rev. Lett. 89, 137007 (2002).

${ }^{31}$ C. Cirillo, S. L. Prischepa, M. Salvato, C. Attanasio, M. Hesselberth, and J. Aarts, Phys. Rev. B 72, 144511 (2005).

${ }^{32}$ K. Matsuda, H. Niwa, Y. Akimoto, T. Uemura, and M. Yamamoto, IEEE Trans. Appl. Supercond. 17, 3529 (2007).

${ }^{33}$ V. N. Kushnir, S. L. Prischepa, J. Aarts, C. Bell, C. Cirillo, and C. Attanasio, Eur. Phys. J. B 80, 445 (2011).

${ }^{34}$ A. A. Golubov, M. Yu. Kupriyanov, and E. Il'ichev, Rev. Mod. Phys. 76, 411 (2004).

${ }^{35}$ F. S. Bergeret, A. F. Volkov, and K. B. Efetov, Rev. Mod. Phys. 77, 1321 (2005). 